\title{
Dynamically reconfigurable high-efficiency terahertz metasurface holograms
}

\author{
Mengyuan $\mathrm{Hu}$ and Zhen Tian ${ }^{*}$ \\ Center for Terahertz Waves and College of Precision Instrument and Optoelectronics Engineering, Tianjin University and the \\ Key Laboratory of Optoelectronics Information and Technology (Ministry of Education), Tianjin 300072, PR China
}

Received: 9 November 2020 / Accepted: 16 December 2020

\begin{abstract}
A reflective, dynamically reconfigurable, high-efficiency metasurface holographic scheme is presented in this paper, which is realized by pumping thin gallium arsenide wafers with a structured femtosecond laser. When the terahertz $(\mathrm{THz})$ passes through the gallium arsenide wafer ( $\mathrm{GaAs})$, the pattern carried by the pump light is converted into the complex permittivity of the light carrier density distribution on the gallium arsenide wafer, which modulates the wafer, thereby changing the transmittance of the $\mathrm{THz}$ wave. The wavefront of the $\mathrm{THz}$ beam is determined by changing the shape and direction of the projected resonator on the DMD by Pancharatnam-Berry (P-B) phase principle. The numerical simulation results show that different holograms can be obtained by dynamically switching the projection on the DMD, and the orthogonal conversion efficiency of circularly polarized light can reach $90 \%$. The holographic scheme proposed in this paper is convenient and fast and may advance the real-time dynamic conversion and dynamic display of holograms.
\end{abstract}

Keywords: Terahertz / metasurface / holograms / P-B phase

\section{Introduction}

Terahertz $(\mathrm{THz})$ technology has potential applications in many fields. In recent years, with the development of terahertz generation and detection technology [1-4], there has been an upsurge of related research on terahertz technology. The development of functional terahertz devices will promote the practical process of terahertz technology, in which spatial light modulators can control the intensity or phase of electromagnetic waves to generate wavefronts of different shapes, which have important applications in imaging, optical information processing, and other fields prospect [5]. In the terahertz frequency range, due to the lack of optoelectronic materials, the development of terahertz spatial light modulators is limited, and the emergence of metasurfaces provides an effective method for it. The metasurface is an artificial metamaterial composed of subwavelength microstructures, it can control the amplitude, phase, and polarization of electromagnetic waves in the subwavelength scale [6-8]. It generally changes the size or orientation of the microstructure [9-11] to control the electromagnetic field. This

\footnotetext{
* e-mail: tianzhen@tju.edu.cn
}

method of using microstructures to locally control the phase of the electromagnetic field can generate almost any wavefront shape.

Holography is a revolutionary three- dimensional imaging technology. Since Gabor [12] first proposed holography in 1948, it has aroused great interest. In 1967, A. W. Lohmann and D. P. Paris [13] proposed computer-generated holography $(\mathrm{CGH})$. CGH generates a hologram through the numerical calculation to obtain the phase distribution at the hologram interface, and encode this information into a specific surface structure, eliminating the need for real objects and highly space-time coherent light sources. The advent of CGH reduces the production cost of holograms and improves the imaging speed of holograms. Traditional CGH usually uses spatial light modulator SLM (such as LCoS and DMD) to generate holograms. Since metasurfaces can control light in the subwavelength range [14-16]. In recent years, metasurfaces have been used to generate holograms [17,18]. Most metasurfaces are composed of structured metal or dielectric resonators. Once they are manufactured by physical means such as photolithography, their encoded information is immutable. If the dynamic modulation problem can be overcome, then metasurfaces can achieve a wider range of applications. At present, the realization of metasurface dynamic modulation mostly uses phase change materials (such as Ge2Sb2Te5 and VO2) [19-22], two-dimensional 
materials (such as graphene and borophene) [23,24], adjustable printed circuits [25], and Liquid metal [26,27]. However, almost all active metamaterials have been applied to the entire structure instead of individually reconfiguring each metasurface unit.

It is still very challenging to simultaneously achieve the low size, high transmission efficiency, real-time continuous reconfigurability on a metasurface. In 2017, reference [28] demonstrated a kind of Huygens Metalens that is actively adjustable at microwave frequencies by applying voltagecontrolled varactor diodes to resonant subatoms, which has fast response time and high efficiency. This work provides unprecedented potential for real-time, fast and complex electromagnetic wave manipulation. In 2019, reference [29] designed a dual-functional metal atom with a comprehensive response to the propagation phase and the geometric phase, and realized an efficient Metalense with switchable functions. In 2020, references [30,31] achieved independent phase control of cross-polarization and co-polarization for circularly polarized waves in metasurface phase wavefront manipulation.

Due to the physical size and control method, it is difficult to control each pixel individually for terahertz waves. In 2019, Professor Zhang Yan's research group [32] proposed a dielectric metasurface based on the P-B phase. The metasurface is realized by pumping thin silicon wafers with a structured femtosecond laser. When changing the pattern carried by the femtosecond laser, different holograms can be dynamically obtained. This method makes it possible to dynamically control the structure of each unit of the metasurface, but due to the low conversion efficiency $(\sim 3.8 \%)$, the holographic imaging effect is not perfect. The defectof the transmissive phase hologram is the low energy utilization, and the reflective metasurface solves this problem well. In 2015, Professor Zhang Shuang's research team [33] designed a hologram with a reflective structure design for rod antennas, which can achieve $80 \%$ efficiency at $825 \mathrm{~nm}$. Here, we designed a reflective high-efficiency P-B phase dielectric metasurface, which can project different resonator shapes on the DMD by pumping beams to excite gallium arsenide, and reconfigure each metasurface unit structure to achieve a dynamics holographic image.

\section{Theory}

\subsection{Principle of Pancharatnam-Berry (P-B) phase}

In 1956, S. Pancharatnam [34] first proposed the concept of geometric phase, and in 1984, Professor V. Berry [35] extended the concept. Then it is called as PancharatnamBerry (P-B) phase. Figure 1 is a schematic diagram of the Poincaré sphere, each point on the surface of the sphere corresponds to a polarization state. When the polarization state of a light wave travels from the north pole to the south pole along the $\mathrm{C} 1$ path, and then back to the north pole along the $\mathrm{C} 2$ path, the phase change is half of the solid angle corresponding to the closed-loop enclosed by the paths $\mathrm{C} 1$ and $\mathrm{C} 2$.

Using Jones calculus [36,37], the reflected field of the P-B element can be calculated for arbitrarily polarized incident wave. For the incident electromagnetic wave of

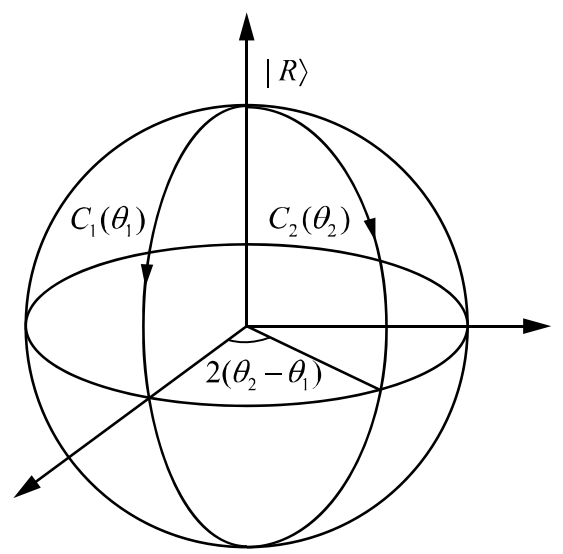

$|L\rangle$

Fig. 1. Poincaré sphere representation of geometric phase.

the left-handed circularly polarized electromagnetic wave, the output electric field after interacting with the reflective metasurface is expressed as

$$
\left[\begin{array}{l}
E_{x} \\
E_{y}
\end{array}\right]=\frac{J_{\zeta}}{\sqrt{2}}\left[\begin{array}{l}
1 \\
i
\end{array}\right]=\frac{1}{\sqrt{2}}\left(\left(r_{u}+r_{v}\right)\left[\begin{array}{l}
1 \\
i
\end{array}\right]+\left(r_{u}-r_{v}\right) e^{2 i \zeta}\left[\begin{array}{l}
1 \\
-i
\end{array}\right]\right)
$$

where $E_{x}$ and $E_{y}$ represents the $x$ and $y$ polarization components of the emitted electromagnetic wave, respectively. $\zeta$ is the direction angle of the metasurface structure $J_{\zeta}$ is the Jones matrix, $r_{u}$ and $r_{v}$ represents the complex amplitude of the fast and slow axis reflections of the metasurface structure, respectively.

According to formula (1), when a left-handed circularly polarized electromagnetic wave is incident on the structure, the excited right-handed circularly polarized electromagnetic wave carries the geometric phase of $2 \zeta$.

\subsection{Gerchberg-Saxton (G-S) phase recovery algorithm}

Gerchberg-Saxton algorithm [38] is used to calculate the required electric field phase distribution on the metasurface. The process is shown in Figure 2 [39].

$$
\begin{aligned}
& U\left(x_{0}, y_{0}\right)=\frac{1}{i \lambda} \iint U(x, y) \cos <n, r>\frac{\exp (i k r)}{r} d x d y \\
& U^{\prime}(x, y)=\frac{1}{i \lambda} \iint U^{\prime}\left(x_{0}, y_{0}\right) \cos <n, r>\frac{\exp (-i k r)}{r} d x_{0} d y_{0}
\end{aligned}
$$

Formula (2) and (3) are the Rayleigh-Sommerfeld diffraction formula and its inverse calculation, respectively, which correspond to the "RS" and "RS ${ }^{-1}$ " operation in Figure 2. $U\left(x_{0}, y_{0}\right)$ and $U(x, y)$ represents the electric field on the image plane and metasurface, respectively. $\lambda$ is the wavelength in vacuum, $\cos \langle n, r\rangle=z / r$ is the tilt factor, and $r=\sqrt{\left(x_{0}-x\right)^{2}+\left(y_{0}-y\right)^{2}}$. 


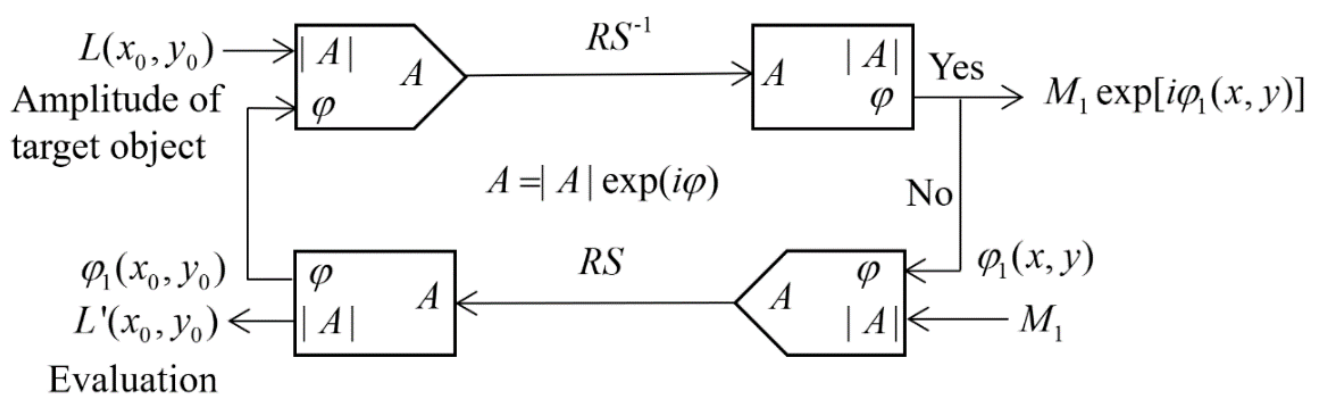

Fig. 2. Gerchberg-Saxton iterative algorithms.

When the error between the amplitude of the reconstructed image and the amplitude of the target image in Figure 2 meets the set error value $\varepsilon$, the iteration is terminated, and $\varphi_{1}(x, y)$ is the desired metasurface phase distribution. Otherwise, the loop calculation continues until the error meets the requirements.

\section{The design of dynamic metasurface holographic structure}

Different from the metasurface produced by traditional physical methods, we project the femtosecond laser onto the encoded DMD and then use the patterned femtosecond laser to pump the thin gallium arsenide wafer. The holographic structure distribution is realized by optical excitation of gallium arsenide. The gallium arsenide wafer is transparent to terahertz waves without any excitation. When light energy is higher than the gallium arsenide band gap $(1.4 \mathrm{eV})$, light carriers are generated on the gallium arsenide surface and extend to the wafer with a penetration depth of about $5 \mu \mathrm{m}$. These carriers attenuate $\mathrm{THz}$ wave transmission by modulating the complex dielectric constant of the wafer. Therefore, the $\mathrm{THz}$ transmittance of projected femtosecond laser on a gallium arsenide wafer decreased while that of unprojected laser remained almost unchanged.

A rectangular column with spatially varying pointing angle is a typical unit structure for constructing P-B phase metasurfaces. Based on the above theory, we designed a reflective dynamic P-B phase dielectric metasurface for 0.5 $\mathrm{THz}$ application. Its unit structure is shown in Figure 3.

Where $l_{x}, l_{y}$ represent the length and width of the beam of the projection unit, respectively. $P_{x}=P_{y}=245 \mu \mathrm{m}$, is the unit period. $t_{1}, t_{2}$ and $t_{3}$ are the thickness of the top thin gallium arsenide film, polyimide film, and bottom aluminum film, respectively, where $t_{1}=10 \mu \mathrm{m}, t_{2}=32 \mu \mathrm{m}$ and $t_{3}=200 \mathrm{~nm}$. The polyimide film acts as a Fabry-Perot cavity, and the bottom metal aluminum film acts as a mirror reflecting incident light, so that the incident lefthanded circularly polarized wave repeatedly oscillates between the top gallium arsenide structure and the bottom metal aluminum film. The reflected beam continues to excite the top gallium arsenide structure and produces an output beam with phase delay, and the polarization conversion is gradually strengthened. Therefore, the conversion efficiency of the reflective structure is higher than that of the transmissive structure.

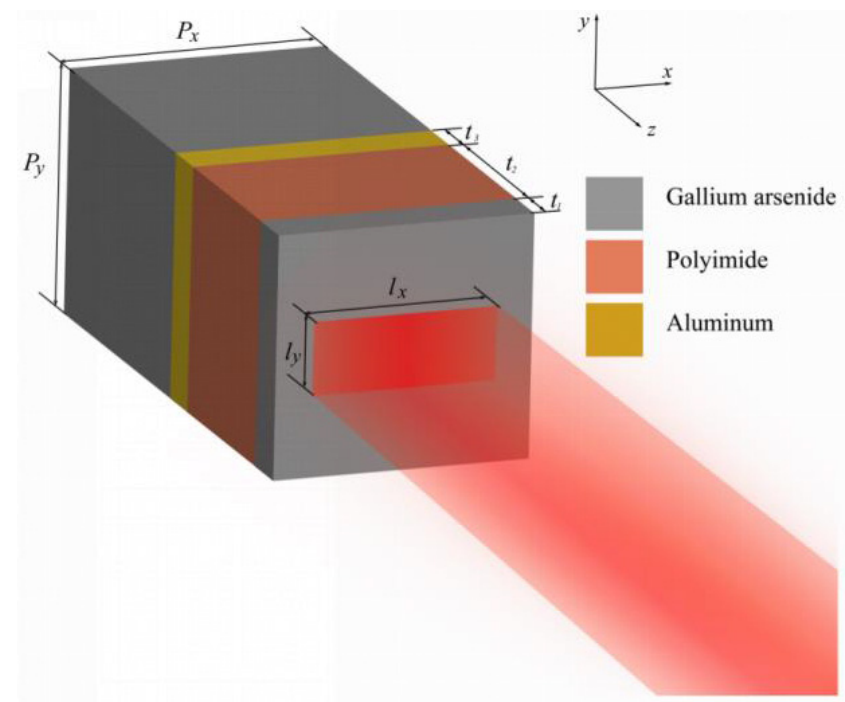

Fig. 3. Schematic diagram of dynamic controllable metasurface unit structure.

To maximize conversion efficiency, optimize the rectangular cell structure parameters using the commercial software, CST Microwave Studio. However, there are no light-excited materials in the library. We know that the conversion efficiency of orthogonal circular polarization depends on the photocarrier density excited in the gallium arsenide column, which in turn depends on the power intensity of the femtosecond pumped laser, and the electrical conductivity of the gallium arsenide semiconductor is positively correlated with the light intensity. According to reference [40], when the light intensity gradually increases from 0 to $60 \mathrm{~J} / \mathrm{cm}^{2}$, more and more valence band electrons in gallium arsenic absorb energy and transition to the conduction band, thus forming a caveelectron pair. Its conductivity rapidly increases from 0 to $10^{6} \mathrm{~S} / \mathrm{m}$. Therefore, in the simulation analysis, the gallium arsenide conductivity can be taken as one of the adjustment parameters, and the variation of light intensity can be simulated and adjusted by adjusting gallium arsenide conductivity.

We first set the conductivity of the gallium arsenide column $\sigma=5000 \mathrm{~S} / \mathrm{m}$, and then use finite difference time domain (FDTD) to perform full-wave numerical simulation in the CST to obtain the best rectangular resonator 
structure size which can convert the incident circularly polarized wave to its orthogonal state with maximum efficiency in $0.5 \mathrm{THz}$. Scan the length and width of the rectangular gallium arsenide rod resonator, the length and width start from 100 and $20 \mu \mathrm{m}$, and scan to 220 and $140 \mu \mathrm{m}$ at $5 \mu \mathrm{m}$ intervals. They form a rectangular array with a period of $245 \mu \mathrm{m}$. The results are shown in Figure 4, which plots the relationship between different length-width combinations and conversion efficiency of gallium arsenide rod resonators. Among them, the maximum conversion efficiency is marked with a white asterisk and its corresponding geometric parameters is $220 \mu \mathrm{m} \times 76 \mu \mathrm{m}$, and all subsequent phase holograms are designed with this geometric parameter.

We use the resonator designed above to explore the relationship between the conductivity of different gallium arsenide at $0.5 \mathrm{THz}$ and the efficiency of circularly polarized light to its orthogonal state.

First, we explored the simulated reflectance coefficient curves of meta-atom under different gallium arsenide conductivity, as shown in Figure 5. In Figure 5a, the conductivity $\sigma=20000 \mathrm{~S} / \mathrm{m}$, and in $5 \mathrm{~b} \sigma=10000 \mathrm{~S} / \mathrm{m}$. The blue solid line and red dashed line are the corresponding cross-polarization and co-polarization amplitudes in the case of incident left-handed circularly

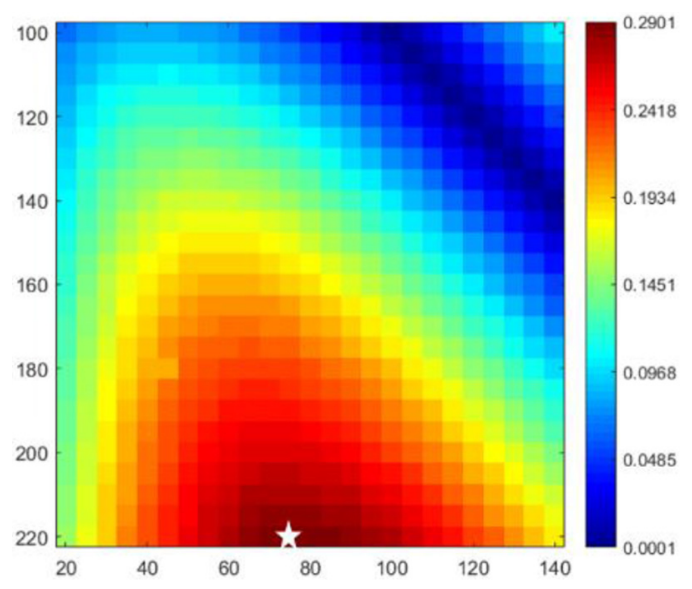

Fig. 4. Parameter optimization of meta-atom.

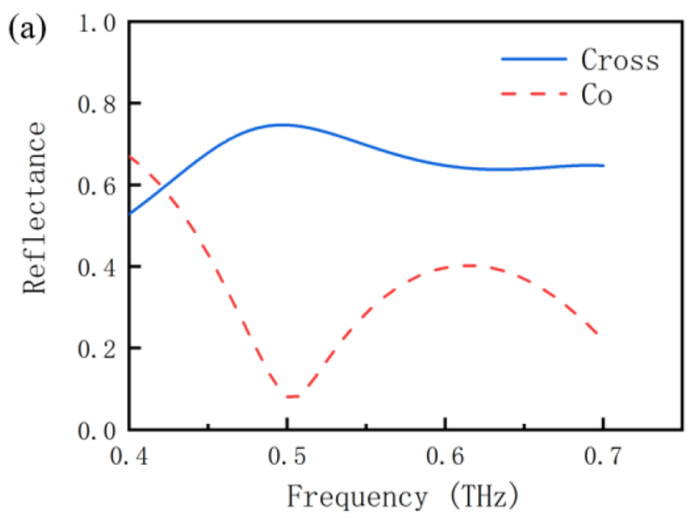

polarized waves. It can be observed that when the working frequency is $0.5 \mathrm{THz}$, most of the incident energy is transmitted in the cross-polarization mode, while the amplitude of the co-polarization is lower than 0.1 , and as the conductivity of the unit structure increases, its orthogonal conversion efficiency has been improved.

To compare the reflection efficiency and the transmission efficiency, we also designed a transmissive metasurface for comparison. The results obtained are shown in Figure 6, the orange dots represent the orthogonal conversion efficiency obtained when the conductivity of gallium arsenide is set as $0,500,1000,2000,3000,5000,10000$, 20000,25000 , and $30000 \mathrm{~S} / \mathrm{m}$ in the simulation, and the blue solid line represents the fitting curve of the orthogonal conversion efficiency with the conductivity of gallium arsenide obtained by the cubic spline function interpolation method on the basis of the above measured values.

Figure $6 \mathrm{a}$ shows the relationship between the conductivity of the transmissive metasurface gallium arsenide and the conversion efficiency of circularly polarized light. With the increase of the conductivity, the conversion efficiency first increases exponentially, then slowly increases, and finally tends to be flat. When the gallium arsenide conductivity $\sigma=5 \times 10^{4} \mathrm{~S} / \mathrm{m}$, the conversion efficiency is about $47 \%$, and then it tends to saturation. Figure $6 \mathrm{~b}$ shows the relationship between the conductivity of the reflective metasurface gallium arsenide and the conversion efficiency of circularly polarized light, its overall trend is the same as that of the transmitted type, but its saturation efficiency is $92 \%$, almost twice as high as that of the transmitted type. When the gallium arsenide conductivity $\sigma=2 \times 10^{4} \mathrm{~S} / \mathrm{m}$, the conversion efficiency is about $90 \%$. Therefore, the designed reflective metasurface structure requires weaker lighting conditions than the transmissive type to reach the near-saturated conversion efficiency state. The simulation results show that reflective metasurface can greatly improve the energy utilization rate.

The P-B scattering phase of orthogonal circular polarization is realized by rotating the orientation of the bar resonator. When the conductivity of gallium arsenide is $\sigma=1 \times 10^{4} \mathrm{~S} / \mathrm{m}$, the simulation results are shown in Figure 7 . The red dashed line and the blue solid line are the geometric phases adjustment results under ideal conditions and simulations, respectively. Orthogonal polarization

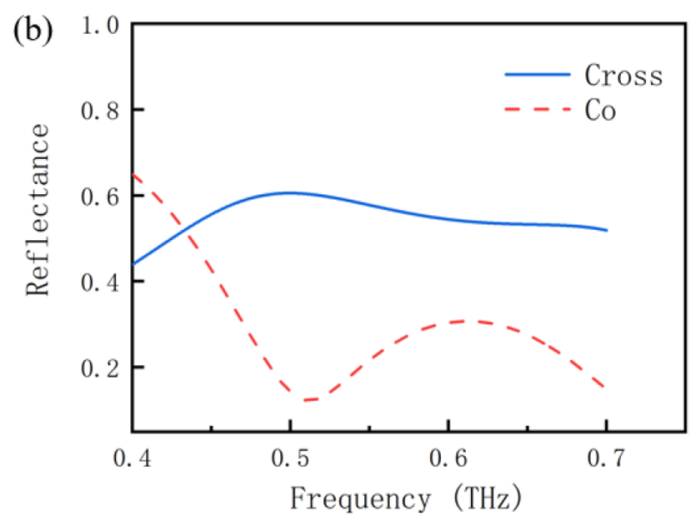

Fig. 5. Simulation reflectance coefficient curve of meta-atoms. Simulation result when (a) $\sigma=20000 \mathrm{~S} / \mathrm{m}$ and (b) $\sigma=10000 \mathrm{~S} / \mathrm{m}$. 
(a)

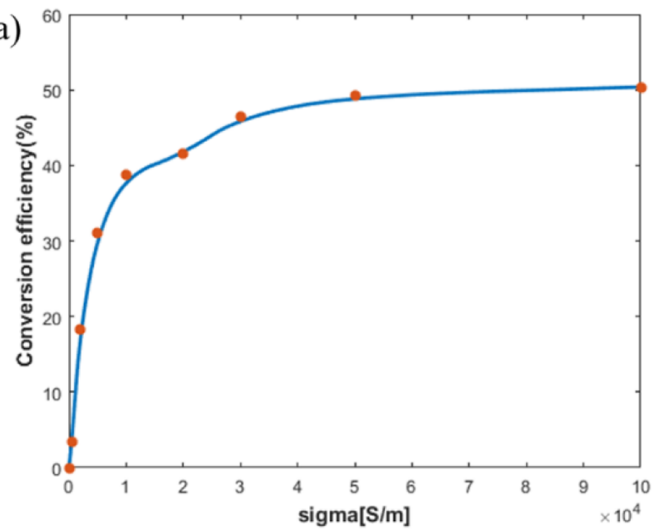

(b)

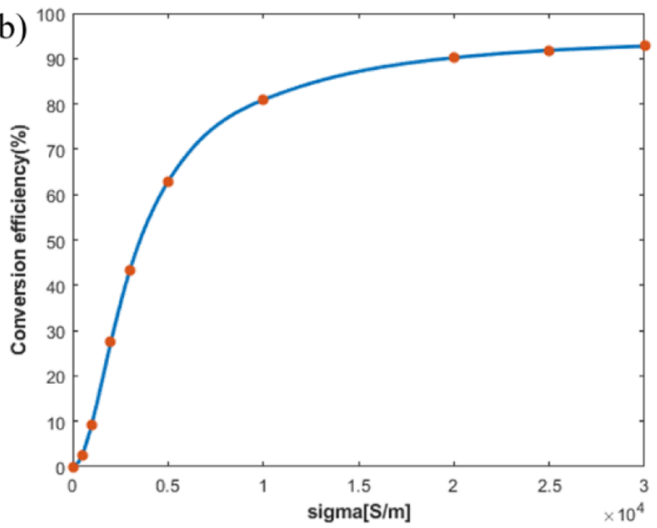

Fig. 6. The relationship between gallium arsenide conductivity and conversion efficiency (a) Transmissive metasurface circularly polarized light conversion efficiency; (b) Reflective metasurface circularly polarized light conversion efficiency.

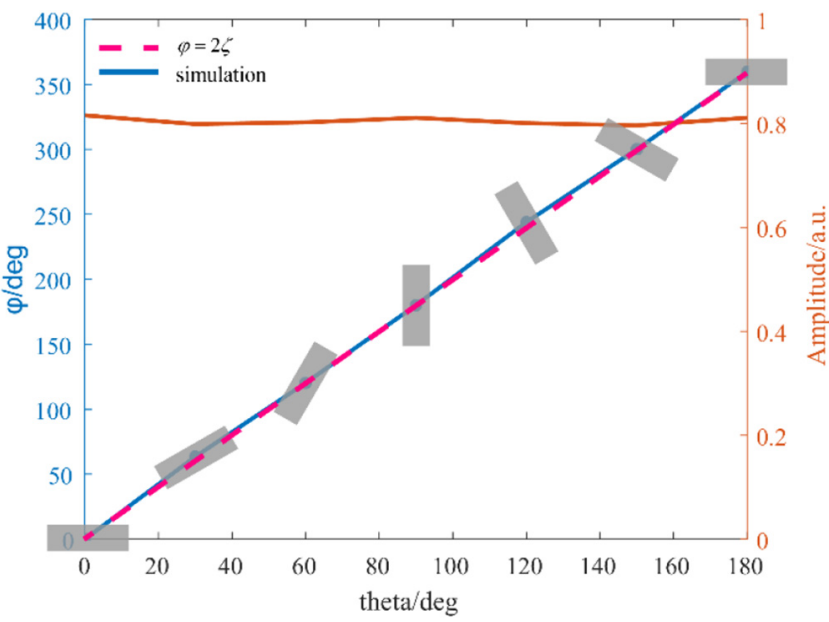

Fig. 7. Simulation effect of geometric phase control of the unit structure.

electromagnetic waves will produce $2 \zeta$ phase mutations related to the structural pointing angle $\zeta$, and the normalized reflection amplitudes obtained from different pointing angles all fluctuate slightly around 0.8. The simulation results are consistent with the theory in the previous section, and the phase control of the unit structure is realized.

\section{The simulation results of dynamic metasurface holographic}

Using the Gerchberg-Saxton phase recovery algorithm mentioned in the previous section to design three metasurface phase holograms applied to $0.5 \mathrm{THz}$. The gallium arsenide conductivity $\sigma=1 \times 10^{4} \mathrm{~S} / \mathrm{m}$ was set in the design to generate the letter "T", "J" and "U", the target image is shown in Figure 8, the size is $14.7 \mathrm{~mm} \times 14.7 \mathrm{~mm}$, the distance between the designed hologram and the metasurface is $z=4 \mathrm{~mm}$. We calculated the phase distribution on the metasurface and normalized the phase of $2 \pi$. The result is shown in Figure 9, which is consistent with the size of the target image region.

After obtaining the metasurface phase distribution, the hologram is encoded into metasurface control parameters using the gallium arsenide metasurface unit structure designed in the previous section. Here, we encode the metasurface into $60 \times 60$ units and change the orientation angle of each unit to achieve the corresponding phase distribution. The resulting "T", "J", "U" metasurface unit structure array is shown in Figure 10.

The electromagnetic wave response characteristics under the orientation angle of each small unit structure in the metasurface structure array are simulated, and then the phase increment obtained by the simulation is brought into the scalar diffraction theory to calculate the electric field distribution on the image plane $4 \mathrm{~mm}$ away from the metasurface. The result is shown in Figure 11. The simulation results show that the letters "T", "J", and "U" are reconstructed. Therefore, the proposed scheme does not need to fabricate a holographic metasurface structure. It only needs to project a preset phase distribution pattern onto the DMD device, and pump the semiconductor with a femtosecond laser carrying the pattern, so that each unit structure of the metasurface can be controlled to achieve a dynamically controllable hologram.

\section{Conclusions}

In this paper, we propose a controllable hologram display method with high conversion efficiency and design the "T", "J" and "U" metasurface phase holograms based on the Gerchberg-Saxton algorithm at $0.5 \mathrm{THz}$, the total efficiency of the simulated holographic image reaches $80 \%$. There is no need to perform complex processing on the metasurface. Changing the pattern information carried by the femtosecond laser can change the gallium arsenide carrier density distribution, leading to changes in the structure of each unit on the metasurface, thereby realizing dynamic control of the metasurface. The simulation results 

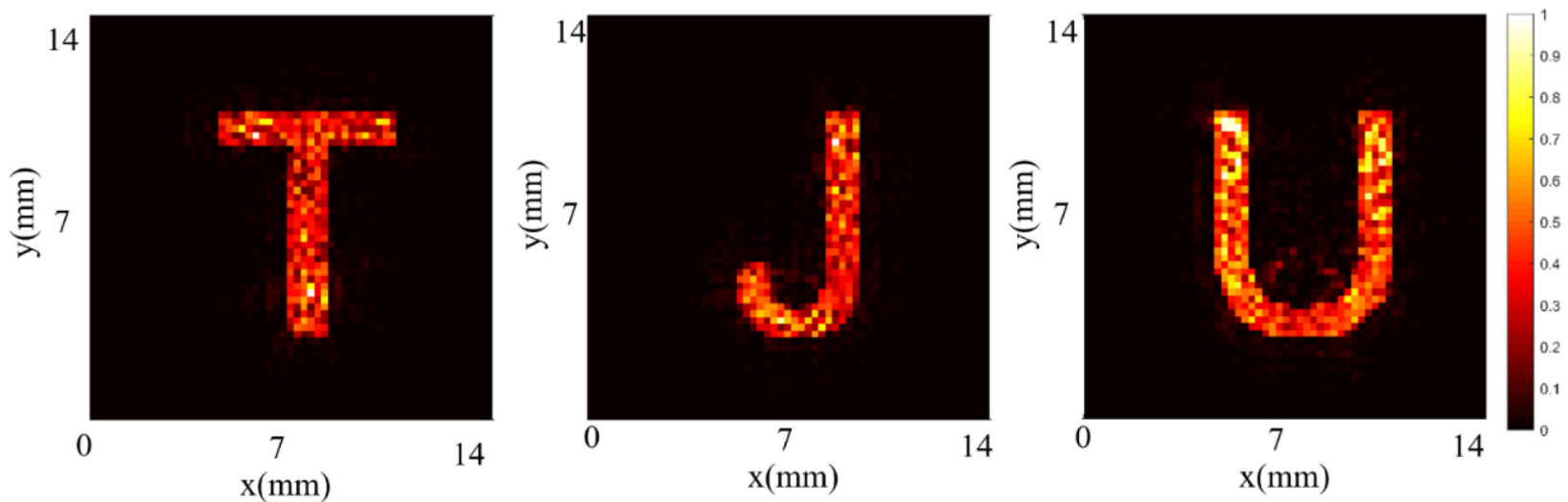

Fig. 8. Amplitude distribution diagram of the designed letters "T", "J" and "U" at the target plane.
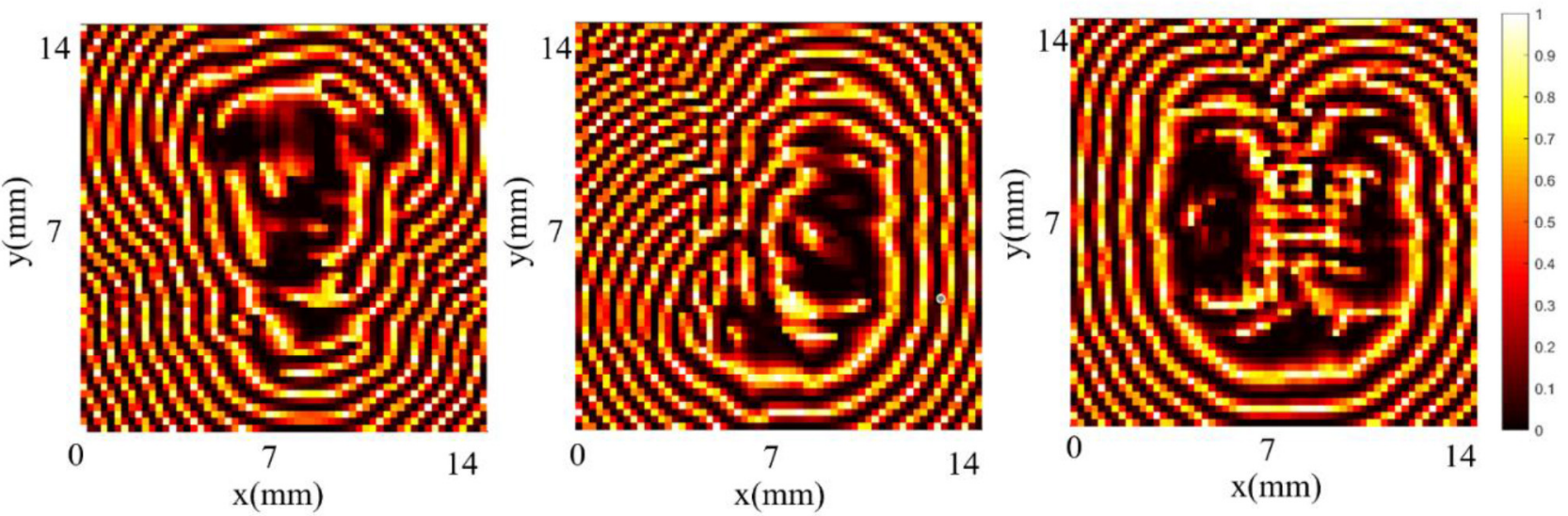

Fig. 9. The metasurface phase distribution of "T", "J" and "U".
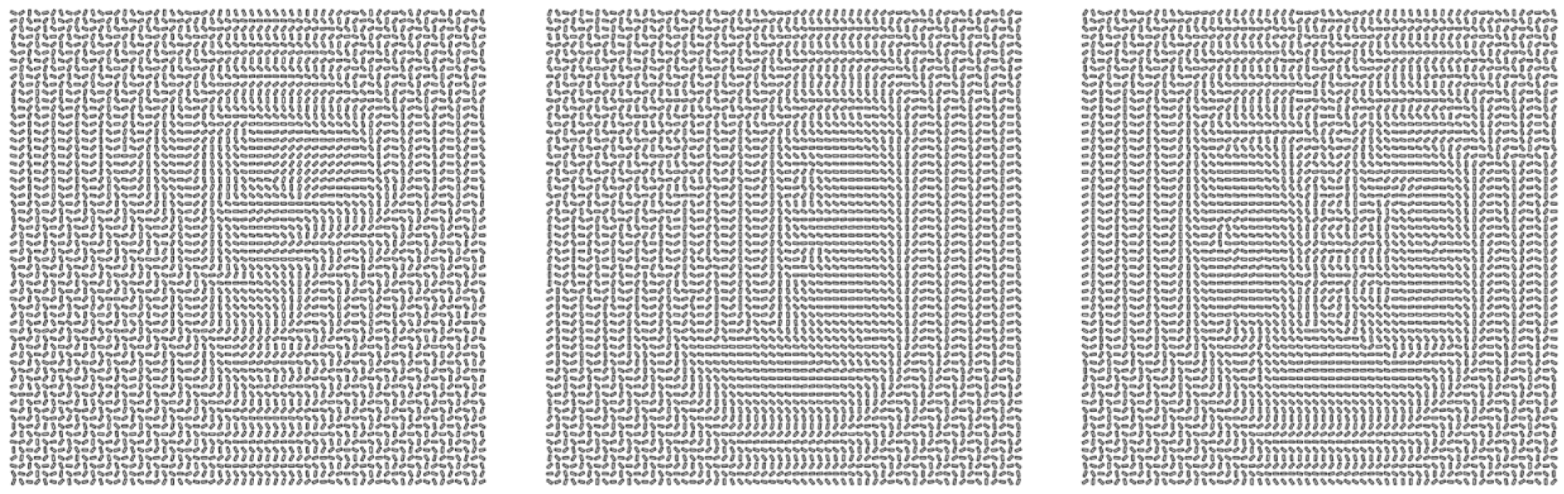

Fig. 10. The metasurface unit structure array of "T", "J" and "U".

show the effectiveness of the proposed reconfigurable $\mathrm{THz}$ metasurface phase hologram, and the efficiency of the circularly polarized light to its orthogonal state is up to $90 \%$. Dynamic holographic control is of great significance to real-time switching and display of holograms. It is worth noting that this work only conducts theoretical and simulation research on terahertz dynamic holographic metasurfaces, so it does not consider the problems that may be encountered in the construction of the experimental system and the specific operational details of the experiment. In future work, we will consider experimental feasibility to design and build a dynamic metasurface imaging system, and improve and optimize the design according to the actual situation. 

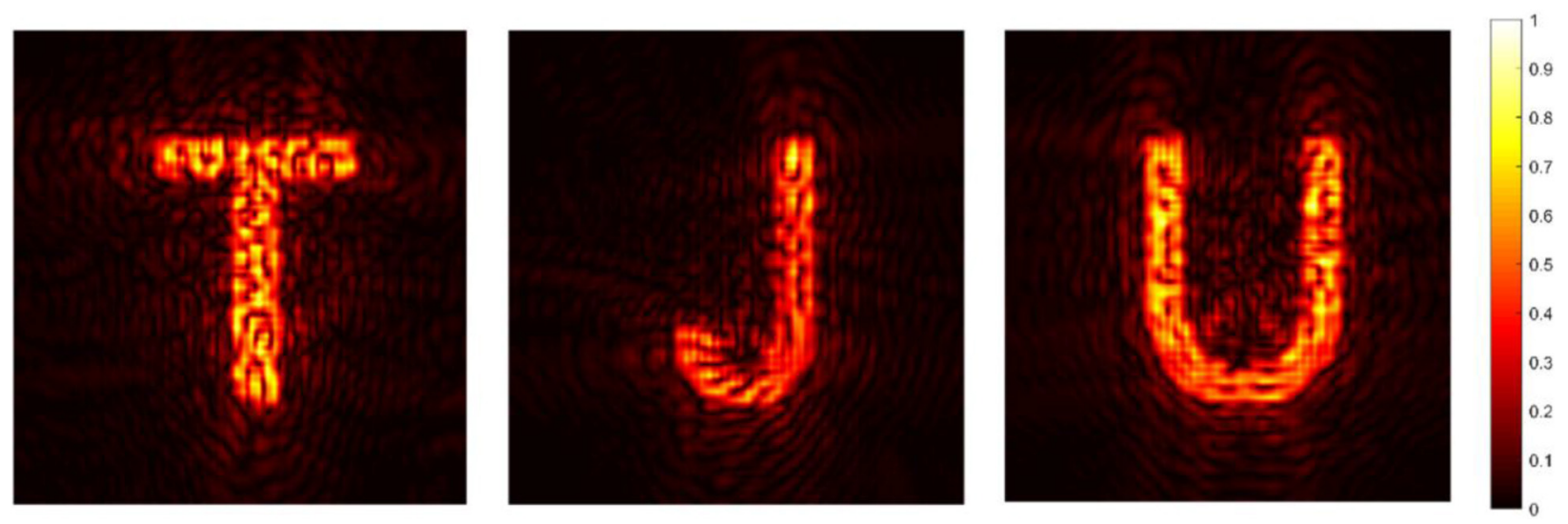

Fig. 11. Field distribution at the target plane of "T", "J" and "U" obtained by simulation.

\section{References}

1. R. Kohler, A. Tredicucci, F. Beltram, H.E. Beere, E.H. Linfield, A.G. Davies, D.A. Ritchie, R.C. Iotti, F. Rossi, Nature 417, 156 (2002)

2. B.S. Willians, Nat. Photonics 1, 517 (2007)

3. C. Belacel, Y. Todorov, S. Barbieri, D. Gacemi, I. Favero, C. Sirtori, Nat. Commun. 8, 1578 (2017)

4. C.G. Wade, N. Sibalic, N.R. de Melo, J.M. Kondo, C.S. Adams, K.J. Weatherill, Nat. Photonics 11, 40 (2017)

5. M. Tonouchi, Nat. Photonics 1, 97 (2007)

6. M. Pu, X. Li, X. Ma et al., Sci. Adv. 1, e1500396 (2015)

7. J. Luo, B. Zeng, C. Wang et al., Nanoscale 7, 18805 (2015)

8. N. Yu, P. Genevet, M.A. Kats et al., Science 334, 333 (2011)

9. J. Zeng, L. Li, X. Yang et al., Nano Lett. 16, 3101 (2016)

10. S. Chen, Y. Cai, G. Li et al., Laser Photonics Rev. 10, 322 (2016)

11. L. Huang, X. Chen, B. Bai et al., Light Sci. Appl. 2, e70 (2013)

12. D. Gabor, Nature 161, 777 (1948)

13. A.W. Lohmann, D.P. Paris, Appl. Opt. 6, 1739 (1967)

14. A. Arbabi, Y. Horie, M. Bagheri, A. Faraon, Nat. Nanotechnol. 10, 937 (2015)

15. X.J. Ni, A.V. Kildishev, V.M. Shalaev, Nat. Commun. 4, UNSP 2807 (2013)

16. L.L. Huang, X.Z. Chen, H. Muhlenbernd, H. Zhang, S.M. Chen, B.F. Bai, Q.F. Tan, G.F. Jin, K.W. Cheah, C.W. Qiu, J.S. Li, T. Zentgraf, S. Zhang, Nat. Commun. 4, 2808 (2013)

17. W.T. Chen, K.Y. Yang, C.M. Wang, Y.W. Huang, G. Sun, I. D. Chiang, C.Y. Liao, W.L. Hsu, H.T. Lin, S. Sun, L. Zhou, A.Q. Liu, D.P. Tsai, Nano Lett. 14, 225 (2014)

18. M.I. Shalaev, J.B. Sun, A. Tsukernik, A. Pandey, K. Nikolskiy, N.M. Litchinitser, Nano Lett. 15, 6261 (2015)

19. A.V. Pogrebnyakov, J.A. Bossard, J.P. Turpin et al., Opt. Mater. Express 8, 2264 (2018)
20. L. Wang, W. Hong, L. Deng et al., Materials 11, 2040 (2018) 21. S.Y. Lee, Y.H. Kim, S.M. Cho et al., Sci. Rep. 7, 41152 (2017)

22. X. Liu, Q. Wang, X. Zhang et al., Adv. Opt. Mater 7, 1900175 (2019)

23. P.C. Wu, N. Papasimakis, D.P. Tsai, Phys. Rev. Appl. 6, 044019 (2016)

24. W. Yao, L. Tang, J. Wang et al., IEEE Photonics J. 10, 4800909 (2018)

25. K. Chen, Y. Feng, F. Monticone et al., Adv. Mater. 29, 1606422 (2017)

26. P.C. Wu, W. Zhu, Z.X. Shen et al., Adv. Opt. Mater. 5, 1600938 (2017)

27. J. Chen, K. Wang, H. Long et al., Nano Lett. 18, 1344 (2018)

28. K. Chen, Y.J. Feng, F. Monticone et al., Adv. Mater. 29, 1606422 (2017)

29. K. Zhang, Y.Y. Yuan, X.M. Ding et al., ACS Appl. Mater. Inter. 11, $28423(2019)$

30. Y.Y. Yuan, K. Zhang, B. Ratni et al., Nat. Commun. 11, 4186 (2020)

31. Y.Y. Yuan, S. Sun, Y. Chen et al., Adv. Sci. 7, 2001437 (2020)

32. J. Guo, T. Wang, H. Zhao et al., Adv. Opt. Mater. 7, 1801696 (2019)

33. G.X. Zheng, H. Muhlenbernd, M. Kenney et al., Nat. Nanotechnol. 10, 308 (2015)

34. S. Pancharatnam, Proc. Natl. Acad. Sci. INDIA A 44, 398 (1956)

35. M.V. Berry, Proc. Roy. Soc. A Math. Phys. 392, 45 (1984)

36. Z. Bomzon, G. Biener, V. Kleiner, E. Hasman, Opt. Lett. 27, 1141 (2002)

37. E. Hasman, V. Kleiner, G. Biener, A. Niv, Appl. Phys. Lett. 82, $328(2003)$

38. R.W. Gerchberg, W.O. Saxton, Optik 35, 237 (1972)

39. Q. Wang, E. Plum, Q.L. Yang et al., Light Sci. Appl. 7, 25 (2018)

40. Z.F. Fan, Z.Y. Tan, W.J. Wan et al., Acta Phys. Sin-CH ED 66, $087801(2017)$ 